\title{
Correction to: Flow shapes and higher harmonics in anisotropic transverse collective flow
}

Danut Argintaru ${ }^{1}$, Calin Besliu ${ }^{2}$, Alexandru Jipa ${ }^{2}$, Tiberiu Esanu ${ }^{4}$, , Valerica Baban ${ }^{1}$, Madalin Cherciu ${ }^{3}$, and Valeriu Grossu ${ }^{2}$

1 Constanta Maritime University, Faculty of Navigation and Naval Transport, Constanta, Romania

2 University of Bucharest, Faculty of Physics, Bucharest, Romania

3 Institute of Space Sciences Bucharest-Magurele, Bucharest, Romania

4 Horia Hulubei National Institute for R\&D in Physics and Nuclear Engineering (IFIN-HH), Magurele, Romania

Original article: Eur. Phys. J. A (2017) 53: 6, https://doi.org/10.1140/epja/i2017-12200-3

Received: 11 September 2017

Published online: 27 September 2017 - C Società Italiana di Fisica / Springer-Verlag 2017

After publication of the paper, the authors realized that the affiliation of the fourth author (Tiberiu Esanu) was given incorrectly. Its correct version appears above.

Moreover, a second funding grant was missed in the acknowledgements. We give their complete correct version below.

This work was supported by the Institute of Atomic Physics-IFA, Romania, under Contracts F09/30.06.2014, Program RO-FAIR, and 09-FAIR/16.09.2016.

\footnotetext{
a e-mail: tiberiu.esanu@nipne.ro
} 